\title{
Leaflet Asymmetry Modeling in Lipid Composition of Escherichia coli Cytoplasmic Membrane
}

Min-Kang Hsieh ${ }^{1}$ and Jeffery B. Klauda ${ }^{1,2^{*}}$

${ }^{1}$ Department of Chemical and Biomolecular Engineering, ${ }^{2}$ Biophysics Program, University of Maryland, College Park, MD 20742, USA

*Corresponding Author: jbklauda@umd.edu

\begin{abstract}
Lipid composition asymmetry between leaflets is important to cell function and plays a key role in the "positive inside" rule in transmembrane proteins. In this work, E. coli inner plasma membrane models reflecting this asymmetry have been investigated at the early-log and stationary stages during the bacterial lifecycle using all-atom molecular dynamics simulations. The CHARMM36 lipid force field is used, and selected membrane properties are tested for variations between two leaflets and whole membranes. Our models include bacterial lipids containing cyclopropane moiety on the sn-2 acyl chain in the stationary membrane. The PE:PG ratio in two leaflets reflects the "positive inside" rule of membrane proteins, set to 6.8 and 2.8 for the inner and outer leaflets of the two models. We are the first to model leaflet asymmetry in lipid composition on E. Coli cytoplasmic membrane and observe the effect on membrane properties in leaflets and whole membranes. Specifically, our results show that for the stationary phase bilayer, the surface area per lipid (SA/lipid) is larger, the thickness $\left(2 D_{C}\right.$ and $\left.D_{B}\right)$ is thinner, the tilt angle is larger, the tilt modulus is smaller, and the deuterium order parameter $\left(S_{C D}\right)$ of $s n-1$ and $s n-2$ tails are lower, compared to the early-log stage. Moreover, the stationary stage bilayer has a negative spontaneous curvature while the early-log stage is near flat spontaneous curvature. For leaflet asymmetry, the inner leaflet has a larger SA/lipid, a smaller thickness, a smaller elastic tilt modulus (a larger tilt angle), and low $S_{C D}$, compared to the outer leaflet in both stages. Moreover, asymmetric membrane involves a lipid tilt and a lateral extension, varying from a reference state of pre-equilibrium membrane. This work encourages a more profound exploration of leaflet asymmetry in various other membrane models and specifically how this might affect structure and function of membrane-associated peptides and proteins.
\end{abstract}




\section{Introduction}

Lipids contain a hydrophilic head group and hydrophobic acyl fatty tails, characterizing an amphiphilic biomolecule, which can self-associate to form bilayers as the main structure of cell membranes at the appropriate water/lipid ratios. As one of the building blocks in a living organism, lipids format the cell membrane and hold transmembrane proteins, having essential roles in the cellular function, i.e., energy storage ${ }^{1}$ and biological signaling ${ }^{2}$, and influence the health state of organisms. ${ }^{3}$ The complexity of biological functions involves local biological events on the membrane and its interactions with other biomolecules that generate a heterogeneous mixture of cellular bilayers, which distributes lipids according to multiple factors, including spontaneously acting of lipid "flip" and presenting of transporters (flippases and floppases), allowing lipids translocation across the bilayer. ${ }^{4}$ For example, negativelycharged phosphatidylserine (PS) is abundant in the inner leaflet of cytoplasmic membrane as an essential co-factor for many membrane proteins, including kinase $\mathrm{C}$ and $\mathrm{Na}^{+} / \mathrm{K}^{+}-\mathrm{ATPase} .{ }^{5}$ Therefore, a feature of cellular membranes is the non-homogeneous distribution of lipids across the bilayer.

Leaflet asymmetry of lipid concentrations have been found in eukaryotic cellular membranes, ${ }^{6-7}$ and essential to their cellular functions. For the red blood cell, the choline-rich phospholipids (phosphatidylcholine and sphingomyelin) have been found in the outer leaflet while phospholipids with a terminal primary amino group, PS, and phosphatidylethanolamine (PE) have been found in the inner leaflet. The changes of outer leaflet causes diseases, such as leukemia ${ }^{8}$ and sickle cell disease. ${ }^{5}$ Loss of membrane asymmetry means the end of the lifespan of the cell. An extremely asymmetric membrane in the bacterial cell is the outer membrane of Gram-negative bacteria, i.e., E. coli, which mainly consisted of phospholipids (PE, phosphatidylglycerol (PG)) in the inner leaflet and lipopolysaccharides (LPSs) in the outer leaflet. ${ }^{9}$ It plays as a selective barrier for survival of bacteria in many distinct environments and resisting to antibiotics. The inner membrane (IM) cellular membrane is also believed to be asymmetrical, ${ }^{10}$ though both leaflets shared the similar lipid types in various concentrations. The bacterial cytoplasmatic membrane almost exclusively consists of PE, PG, and cardiolipin lipids. Bacterial lipids, which contain a cyclopropane moiety on the acyl fatty tails at the 9-10 carbon position, have been found in many bacteria, including E. coli. ${ }^{11-12}$ The amount of this unique lipid depends on the different stages of the bacterial life cycle, i.e., the cyclopropane moiety increases from $10 \%$ to $70 \%$ of acyl chains with the change of colony life cycle of $E$. Coli from early-log to overnight stages.

Modeling and molecular simulations of multiple-component lipid bilayers closer to the natural membranes concentrations has been a recent focus, due to the increase in computational power that 
allows for longer simulations with larger system required for these membrane models. ${ }^{13-14}$ The advanced bilayer models having lipid diversity in membrane beyond two lipid components allow to simulate of a more realistic cell membrane of yeast, ${ }^{15-16}$ P. aeruginosa ${ }^{17}$ and E. Coli. ${ }^{18-20}$ The cytoplasmic or inner membrane (IM) of E. Coli has a diverse lipid head groups including $70-80 \%$ PE, $10-20 \%$ PG, and 0-10\% cardiolipin. ${ }^{21-24}$ The initial lipid diversity models for the bacterial IM consist of bilayers of POPE and POPG to match the ratio of lipid head groups. While the lipid head group diversity has been included in these models, they ignore the lipid chain diversity. Then, the first complex bacterial IM models, which consist of a bilayer including both head group and acyl chain diversity, were proposed in our lab. The top6 membrane model, ${ }^{18}$ which represents the lipid composition of the overnight stage, was developed with a 4.2 PE:PG ratio and including various fatty acid chains of 15:0, 16:0, 16:1, 18:1, and cyC17:0 in 7\%, 40\%, $8 \%, 10 \%$, and $35 \%$, respectively. Later, four stages of the bacterial lifecycle models ${ }^{19}$ were constructed based on these diverse lipids with the same PE:PG ratio and emphasizing different percentage of cyC17:0 acryl chain at $0 \%, 9 \%, 26 \%$, and 35\% representing early-log, midlog, stationary, and overnight stages, respectively. These studies more accurately reflected the devise lipid population within $E$. coli cytoplasmic membrane. They revealed that the complex multi-component lipid membrane models have a lower surface density of the bilayer and more rigid than simple two-component POPE/POPG bilayer due to the inclusion of the cyclopropane ring in the acyl chain. The various lipid chain length produces a thinner bilayer with a better agreement with the hydrophobic thickness of known E. Coli transmembrane proteins. We believe that the multi-component bilayer models with diverse lipids more accurately mimic the bacterial membranes than earlier models.

This work simulates a more realistic membrane of organisms with the natural asymmetric distribution of its lipid concentration between two leaflets. The OM of $E$. coli, a typical example of leaflet asymmetry, has been studied recently in our group in collaboration with Wonpil Im. ${ }^{25}$ Models of asymmetric OM of E. Coli have been constructed that consisted of LPS outer leaflet and phospholipids inner leaflet, studying the membrane interaction with $\mathrm{OmpLA}^{20}$ and exploring physical properties of the membrane with neutron reflectionmetry. ${ }^{25}$ Unlike the OM of $E$. coli, the IM leaflets have the same head groups of lipids with a slight difference in lipid composition. The small change should vary depending on the biological activities at the representing bilayers. We construct $E$. coli bacterial cytoplasmic membrane to model the bilayer near a transmembrane (TM) protein insertion region. According to the "positive inside" rule ${ }^{26}$ of TM proteins, that is, the cytoplasmic loops of integral membrane proteins tend to have a greater number of cationic residues than extracellular loops, a corresponding lipid distribution should have more negative charge in the inner leaflet of the IM. This work would investigate the change of membrane properties 
reflecting the leaflet asymmetry in composition and served as a foundation for further studies in a more complex bilayer environment involving other biological molecules, such as TM proteins.

\section{Methods}

This work aims to simulate the lipid concentration asymmetry between leaflets of $E$. Coli cytoplasmic membrane. The model membranes are based on the developed multi-component bilayer models of the top $6^{18}$ and four bacterial life cycle stages. ${ }^{19}$ The former was one of the first lipid diversity models include a cyclic moiety in lipid tail; the latter was based on experimental data of lipid composition of bacterial IM membranes during the developing bacterial lifecycle. One major challenge is the lack of accurate experimental data for the lipid concentration for the individual leaflets, so that the model membrane requires an educated guess of the lipid concentration. Table 1 shows the amount of lipid components for the asymmetric bilayers. Two lipid distributions across the membrane leaflets have been set to simulate the early-log and stationary stages of the lifecycle, respectively. According to the positive-inside rule, our lipid asymmetric membrane models have a more negative charge on the inner leaflet than the outer leaflet. Specific, PE/PG ratio is set to 6.8 at the outer leaflet and 2.8 at the inner leaflet in both stages, compared to the previous symmetric leaflet model at 4.1. Moreover, the distribution of cyclopropane moiety also varies depending on the stage of the lifecycle. In the stationary stage, the bacterial-specific lipids having a cyclopropane moiety, i.e., PMPE, QMPE, and PMPE have been included in a total $25.6 \%$ of acyl tails, compared to the previous model at $26.3 \%$. The cyclic tail in the outer leaflet is $27.6 \%$ which is slightly higher than the inner leaflet (23.7\%). There are no cyclopropane tails in the early log stage in the model. A total of 154 lipids have been simulated in each asymmetric membrane, including 78 and 76 lipids at the outer and inner leaflets, respectively; this difference of lateral lipid numbers could potentially introduce a slightly curve in the membranes, which was observed in the tilt angles. Although it has been suggested that the inner bacterial membrane contains $<10 \%$ cardiolipin, located in the polar and septal regions, our models did not include cardiolipin lipid to represent the membrane away from these curved regions. 
Table 1. Lipid Composition of simulated membranes and the previous model membranes are also shown for comparison.

\begin{tabular}{|c|c|c|c|c|c|c|c|}
\hline \multirow[b]{3}{*}{$\begin{array}{l}\text { Model } \\
\text { lipid }\end{array}$} & \multirow[b]{3}{*}{ sn-1/sn-2 } & \multicolumn{4}{|c|}{$\begin{array}{c}\text { This work } \\
\text { (asymmetric) }\end{array}$} & \multicolumn{2}{|c|}{$\begin{array}{l}\text { Previous model }{ }^{19} \\
\text { (symmetric) }\end{array}$} \\
\hline & & \multicolumn{2}{|c|}{ Early-log } & \multicolumn{2}{|c|}{ Stationary } & \multirow{2}{*}{$\begin{array}{c}\text { Early-log } \\
\text { Leaflet }\end{array}$} & \multirow{2}{*}{$\begin{array}{c}\text { Stationary } \\
\text { Leaflet }\end{array}$} \\
\hline & & $\begin{array}{l}\text { Outer } \\
\text { leaflet }\end{array}$ & $\begin{array}{l}\text { Inner } \\
\text { leaflet }\end{array}$ & $\begin{array}{l}\text { Outer } \\
\text { leaflet }\end{array}$ & $\begin{array}{l}\text { Inner } \\
\text { leaflet }\end{array}$ & & \\
\hline POPE & $16: 0 / 18: 1$ & 12 & 12 & 6 & 6 & 12 & 6 \\
\hline PYPE & $16: 0 / 16: 1$ & 50 & 38 & 17 & 17 & 45 & 17 \\
\hline OYPE & $18: 1 / 16: 1$ & 6 & 6 & 5 & 5 & 6 & 5 \\
\hline PYPG & $16: 0 / 16: 1$ & 10 & 20 & 7 & 12 & 15 & 9 \\
\hline PMPE & $\begin{array}{c}16: 0 / 17: 0 \text { сус } \\
(9,10)\end{array}$ & 0 & 0 & 32 & 20 & 0 & 27 \\
\hline QMPE & $\begin{array}{c}15: 0 / 17: 0 \text { cyс } \\
(9,10)\end{array}$ & 0 & 0 & 8 & 8 & 0 & 8 \\
\hline PMPG & $\begin{array}{c}16: 0 / 17: 0 \text { cyс } \\
(9,10)\end{array}$ & 0 & 0 & 3 & 8 & 0 & 6 \\
\hline total & & 78 & 76 & 78 & 76 & 78 & 78 \\
\hline
\end{tabular}

Pre-equilibrium bilayers

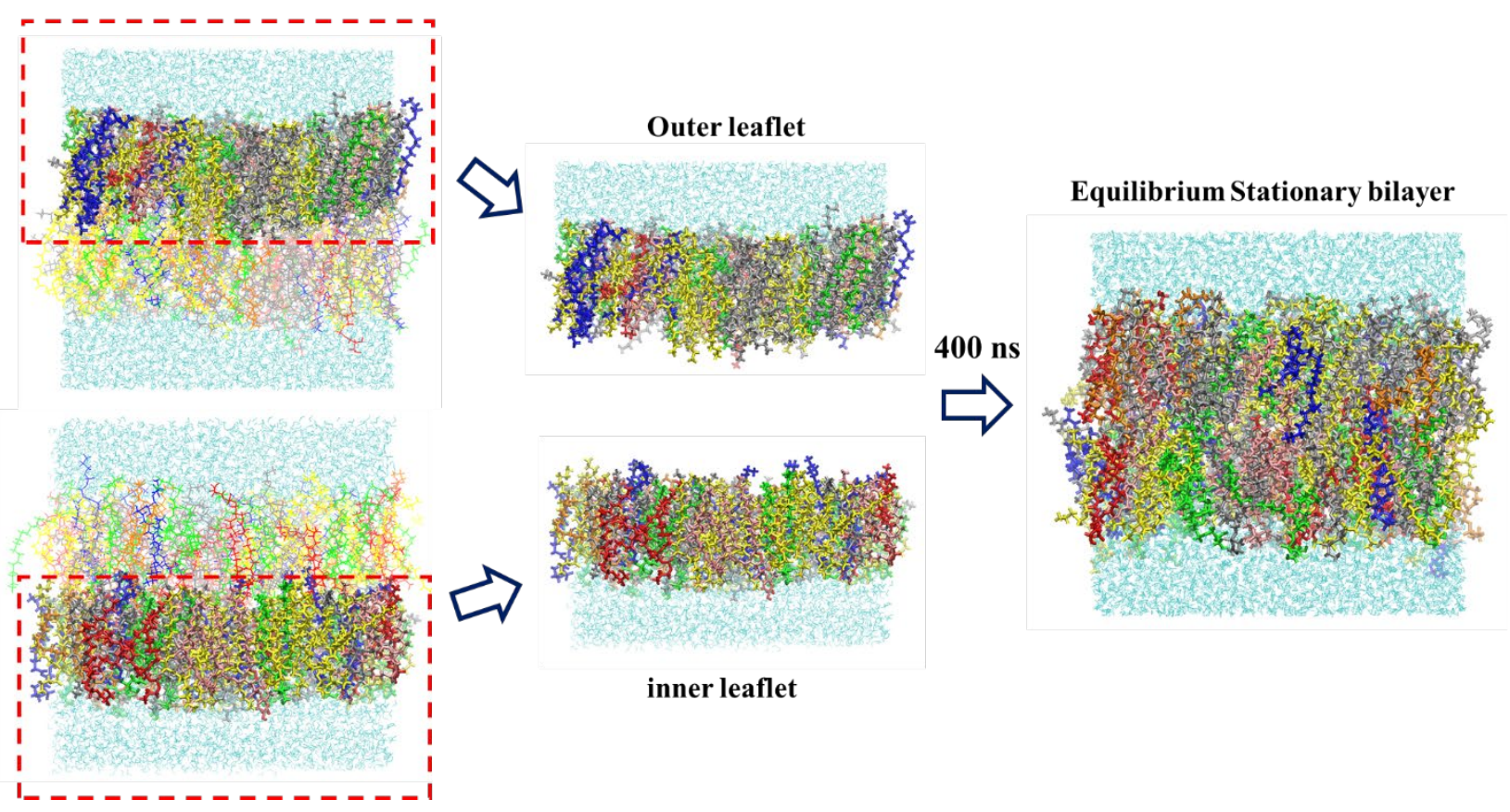

Figure 1. Construction of asymmetric bilayer as follows: extracting the pre-equilibrium configurations of lipid leaflets from two individual symmetric bilayers, then combining the selected leaflet configurations with their lateral waters to form an asymmetric membrane, followed by adding the naturalizing ions. Energy minimization was applied before a $400 \mathrm{~ns}$ production run. The procedure was processed by 
modifying the CHARMM-GUI Membrane builder codes. POPE in blue, PYPE in yellow, OYPE in orange, PYPG in green, PMPE in silver, QMPE in pink, PMPG in red, and water in cyan.

As described above, the construction of our asymmetric bilayers (Figure 1) requires pre-equilibrium of symmetric bilayers to provide the samples of equilibrium leaflets with each desired lipid composition. We have developed a tool to combine any equilibrium leaflets, even in different sizes, and though this work demonstrated its capability to combine leaflet configurations at a similar size. The pre-equilibrium symmetric outer and inner bilayers were processed to extract the equilibrium configurations of lipid leaflets, then the selected leaflet configurations are combined to form an asymmetric membrane. Table 2 shows the size and parameters of both symmetric and asymmetric model membranes. The initial lipid conformations of the pre-equilibrium symmetric membranes were constructed using CHARMM-GUI Membrane Builder ${ }^{16,27-30}$ with a selected lipid composition (Table 1). The simulation box also includes explicit water in the ratio 35:1 of water molecules to lipids and naturalizing ions. For asymmetric membranes, the lateral waters corresponding to the selected leaflets were also extracted to keep the equilibrium of molecular association between the lipids and waters surrounding the leaflets; therefore, the number of waters from each equilibrium configuration varies in each asymmetric membrane, but systems roughly keep the water:lipid ratio at 35 . The neutralizing ions were randomly re-assigned for each asymmetric bilayer in this work using a faster distance-based algorithm, ${ }^{29}$ which the ions were placed near membrane surfaces and avoid bad contacts with other heavy atoms within $4 \AA$. Three replicas for each bilayer composition were carried out to obtain better statistics. Both symmetric and asymmetric MD simulations were conducted using NAMD package ${ }^{31}$ with the CHARMM36 (C36) force field ${ }^{32}$ and the modified TIP3P water model. ${ }^{33-34}$ Parameters for the cyclic moiety on lipid tails were developed by Pandit and Klauda. ${ }^{18}$ The isothermal-isobaric ensemble (NPT) ensemble at $310.15 \mathrm{~K}$ and $1 \mathrm{~atm}$ was used. The Lennard-Jones (L-J) potential was used to describe van der Waals interactions, and a force-based switching function in the range of 8-12 $\AA$ was chosen..$^{35}$ Langevin dynamics maintained the temperature, and the Nosé-Hoover Langevin piston algorithm ${ }^{36-37}$ was applied to maintain the pressure. Hydrogen atoms were constrained by using the RATTLE algorithm. ${ }^{38}$ Simulations were run for $400 \mathrm{~ns}$ with $2 \mathrm{fs}$ time step. At this simulation length, lipids flip across the membrane were not observed. 
Table 2. System size and parameters for each membrane.

\begin{tabular}{|c|c|c|c|c|c|c|c|}
\hline Model & $\begin{array}{l}\text { Total } \\
\text { lipids }\end{array}$ & $\begin{array}{c}\text { Number of } \\
\text { waters* }\end{array}$ & $\begin{array}{c}\text { Number of } \\
\mathrm{K}+\end{array}$ & $\begin{array}{l}\% \text { acyl tail } \\
\text { with cyc17 }\end{array}$ & $\begin{array}{c}\% \text { unsaturated } \\
\text { tail }\end{array}$ & $\begin{array}{c}\mathrm{PE}: \mathrm{PG} \\
\text { ratio }\end{array}$ & Total atoms* \\
\hline \multicolumn{8}{|c|}{ asymmetric membranes } \\
\hline early-log & 154 & $5369-5391$ & 30 & 0 & 53.9 & 4.1 & $34715-34781$ \\
\hline stationary & 154 & $5310-5395$ & 30 & 25.6 & 27.6 & 4.1 & $34647-34902$ \\
\hline \multicolumn{8}{|c|}{ pre-equilibrium membranes } \\
\hline earlylog-outer & 156 & 5460 & 20 & 0 & 53.8 & 6.8 & 35196 \\
\hline earlylog-inner & 152 & 5320 & 40 & 0 & 53.9 & 2.8 & 34360 \\
\hline stationary-outer & 156 & 5460 & 20 & 27.6 & 25.6 & 6.8 & 35326 \\
\hline stationary-inner & 152 & 5320 & 40 & 23.7 & 29.6 & 2.8 & 34448 \\
\hline
\end{tabular}

"number of waters and total atoms of asymmetric membranes vary in replicas because number of lateral waters with pre-equilibrium leaflets are different. A range of number among replicas are provided.

Various analysis is performed to investigate the effect of the leaflet asymmetry in lipid composition at the early-log and stationary stages of $E$. coli cytoplasmic membrane. The surface area per lipid (SA/lipid) of bilayers has been utilized to examine and confirm the equilibrium of all simulation systems. It is evaluated by the area of $x-y$ plane of the simulation box divided by the number of lipids of each leaflet. The area compressibility modulus, $K_{A}$, was calculated as,

$K_{A}=\frac{k_{B} T\langle A\rangle}{\sigma_{\langle A\rangle}^{2}}$

where $k_{B}$ is the Boltzmann constant, $\langle A\rangle$ is the average surface area, $\mathrm{T}$ is temperature, and $\sigma_{\langle A\rangle}^{2}$ is the variance of the area. The elastic tilt modulus per lipid, $K_{t}$, was calculated as ${ }^{39}$

$K_{t}=\frac{k_{B} T}{A_{L}\left\langle t^{2}\right\rangle}$

where $\left\langle A_{L}\right\rangle$ is the average surface area per lipid (or SA/lipid) and $t$ is the tilt, defined as

$$
t=\frac{n}{n \cdot N}-N
$$

where $\boldsymbol{n}$ is the hydrophobic chain vector (from C1 to the end methyl carbon) and $\boldsymbol{N}$ is the leaflet normal. The scale of the tilt vector is equal to the $\tan \theta_{t}$, and $\theta_{t}$ is the tilt angle of hydrophobic chain (the angle between $\boldsymbol{n}$ and $\boldsymbol{N}$ ). Comparing with monolayers having the same composition in both symmetric and 
asymmetric membranes, the relation among the tilt modulus, the surface area per lipid, and thickness shows that the modeled asymmetric membranes involve a lipid tilt and lateral stretching deformation.

The electron density profile (EDP) along the bilayer normal axis (z-axis) was calculated to evaluate the bilayer thickness $\left(D_{B}\right)$, hydrophobic core thickness $\left(2 D_{C}\right)$, and head-to-head group distance $\left(D_{H H}\right)$. The $D_{B}$ is defined as the distance between the midpoints of the water EDP; the $2 D_{C}$ is defined as the distance between the midpoints of the hydrophobic tail EDP; and the $D_{H H}$ is defined as the distance between the peaks of the total EDP. To obtain the EDPs of model membranes, the center of geometry of bilayer was fixed at $Z=0$, allowing the density profiles of both top and bottom leaflets to present symmetrically around $Z=0$. For instance, the minimum intensity of total EDP (or the midplane) located at $Z=0$ for symmetric bilayers. However, the center of geometry of bilayer and the minimum intensity in total EDP mismatches when the same process applied to asymmetric membranes (i.e., the minimum intensity in total EDP locates at around $Z=-0.2 \AA$ in our models). A quadratic curve has been used to fit the basin region of total EDPs to determine the minimum point of total EDP, where is defined as the midplane of the membrane. The leaflet thicknesses were calculated based on the distance between the midplane and the lateral position defined as the mentioned previously. The interdigitation of lipids was obtained from the EDPs for both leaflets as follows: ${ }^{40}$

$\lambda=4 \int_{-L / 2}^{+L / 2} \frac{\rho_{\text {inner }}(z) \times \rho_{\text {outer }}(z)}{\left(\rho_{\text {inner }}(z)+\rho_{\text {outer }}(z)\right)^{2}} d z$

where $\lambda$ is the degree of interdigitation, $\rho_{\text {inner }}$ and $\rho_{\text {outer }}$ are the densities of the inner and outer leaflets at distance $z$ from the center of bilayer, and the integral range $[-L / 2, L / 2]$ is non-zero density along $Z$ axis.

The pressure profile along the z-axis was calculated to evaluate the surface tension $(\gamma)$ and spontaneous curvature $\left(F^{\prime}(0)\right)$ of bilayer ${ }^{41-42}$. The surface tension of monolayer was obtained as follows:

$\gamma=\int_{0}^{\frac{L}{2}}\left[P_{T}(z)-P_{N}\right] d z$

where $P_{T}$ and $P_{N}$ are the tangential and normal components of pressure tensor. The integral range is from the midplane of bilayer $(z=0)$ to the bilayer surface $(z=L / 2)$ for both the inner and outer leaflets of the bilayer. Note that, for a none applied tension bilayer, the surface tension of the inner and outer leaflets is the same absolute value but opposite signs (i.e., $\gamma_{\text {inner }}+\gamma_{\text {outer }}=0$ ) which has been observed in all simulations. To probe the leaflet asymmetry induced bending of bilayer, the first derivative of bending free energy per lipid at zero curvature was calculated for each leaflet to access the energetically favorable bending, ${ }^{41}$ as follows: 
$F^{\prime}(0)=\left.\frac{d F(0)}{d c}\right|_{c=0}=-\int_{0}^{\frac{L}{2}} z\left[P_{T}(z)-P_{N}\right] d z=-K_{C}^{m} C_{0}$

where $F^{\prime}(0)$ is the first derivative of bending free energy per lipid at zero curvature of monolayer; $K_{C}^{m}$ is the monolayer bending constant and $C_{0}$ is spontaneous curvature. The spontaneous curvature could be obtained once the bending constant is determined. Here, the $F^{\prime}(0)$ of individual leaflets in the bilayer were used to access the bending tendency (the direction or spontaneous curvature). The pressure profiles were carried out by analyzing the extended simulation runs beginning from four checked points (every $25 \mathrm{~ns}$ in the last 100 ns of production runs). Each extended run using the particle-mesh Ewald method for the electrostatic interaction with a cutoff of $12 \AA$, a $\kappa$ value of 0.32 and an L-J function for the van der Waals dispersion term with a force-switching function between 8 to $12 \AA$ simulated for 500 ps with the NTP ensemble at $310.15 \mathrm{~K}$ and $1 \mathrm{~atm}$ with $1 \mathrm{fs}$ time step. ${ }^{41}$

The deuterium order parameters of acyl chain $\left(S_{C D}\right)$ were calculated by the defined formula,

$S_{C D}=\left\langle\frac{3}{2} \cos ^{2} \beta-\frac{1}{2}\right\rangle$

where $\beta$ is the angle between the $\mathrm{C}-\mathrm{H}$ vector and the bilayer normal vector. The two-dimensional radial distribution function (2D-RDF) is calculated based on the coordinate of phosphates within PE-PE, PE-PG, and PG-PG pairs, and the lipid-lipid pair represents the average for all lipid pairs. Above analysis applies to individual leaflet as well as a whole bilayer to probe the lateral and overall membrane properties.

\section{Results}

The equilibrium SA/lipid (Table 3 ) is obtained using the Pymbar package ${ }^{43}$ to analyze the time series of SA/lipid (Figure S1), and the result indicated all simulation bilayers reach equilibrium very quickly within 200 ns. The SA/lipid of the inner and outer leaflets in the asymmetric bilayer is $60.86 \pm 0.08$ and $62.46 \pm$ $0.08 \AA^{2}$, respectively, at the early-log stage and $62.41 \pm 0.09$ and $64.05 \pm 0.10 \AA^{2}$ at the stationary stage. Comparison with the symmetric bilayers, the SA/lip of each leaflet in asymmetric membrane is larger, except for the outer leaflet at the stationary stage (Table 3). The comparison indicates the lipids have significantly adjusted their surface area in asymmetric membranes. It has been known that bilayers containing cyclopropanes and PG headgroups have a larger SA/lipid, and our simulations follow this general tendency. The overall average surface area per lipid of asymmetric bilayers for the early-log and stationary stages is $61.66 \pm 0.08$ and $63.23 \pm 0.10 \AA^{2}$, respectively, similar to the previous simulation of 
bacterial lifecycle models ${ }^{19}$ that was $60.77 \pm 0.02 \AA^{2}$ and $62.48 \pm 0.07 \AA^{2}$ at the early-log and stationary stages, respectively.

Table 3. Surface area per lipid, surface compressibility modulus, tilt angle and tilt modulus of model membranes.

\begin{tabular}{ccccc}
\hline \hline Model & $\mathrm{SA} /$ lipid $\left(\AA^{2}\right)$ & $\mathrm{K}_{\mathrm{A}}(\mathrm{N} / \mathrm{m})$ & $\theta_{\mathrm{t}}$ (degree) & $\mathrm{K}_{\mathrm{t}}(\mathrm{mN} / \mathrm{m})$ \\
\hline earlylog membrane & $61.66 \pm 0.08$ & & & \\
outer leaflet & $60.86 \pm 0.08$ & $0.26 \pm 0.01$ & $29.27 \pm 0.09$ & $22.29 \pm 0.18$ \\
inner leaflet & $62.46 \pm 0.08$ & $0.26 \pm 0.01$ & $30.55 \pm 0.08$ & $19.58 \pm 0.15$ \\
stationary membrane & $63.23 \pm 0.10$ & & & \\
outer leaflet & $62.41 \pm 0.09$ & $0.29 \pm 0.01$ & $29.88 \pm 0.18$ & $20.67 \pm 0.33$ \\
inner leaflet & $64.05 \pm 0.10$ & $0.29 \pm 0.01$ & $31.38 \pm 0.15$ & $17.86 \pm 0.24$ \\
symmetric membranes & & & & \\
earlylog-outer & $60.18 \pm 0.10$ & $0.26 \pm 0.02$ & $28.88 \pm 0.07$ & $23.24 \pm 0.14$ \\
earlylog-inner & $61.20 \pm 0.05$ & $0.26 \pm 0.01$ & $29.55 \pm 0.09$ & $21.66 \pm 0.15$ \\
stationary-outer & $62.76 \pm 0.04$ & $0.31 \pm 0.01$ & $30.36 \pm 0.08$ & $19.78 \pm 0.13$ \\
stationary-inner & $63.46 \pm 0.02$ & $0.29 \pm 0.01$ & $30.84 \pm 0.04$ & $18.81 \pm 0.05$ \\
\hline \hline
\end{tabular}

The area compressibility modulus, $0.26 \pm 0.01 \mathrm{~N} / \mathrm{m}$ in the early-log membrane and $0.29 \pm 0.01 \mathrm{~N} / \mathrm{m}$ in the stationary membrane, is nearly identical to that of the symmetric membranes (Table 3 ). However, the $K_{A}$ difference between the two stages is insignificant according to its P-value (Table S1), which disagrees with the previous symmetric membrane models for the bacterial lifecycle that reported statistically significant differences in $K_{A}$ among the different stages of the lifecycle. ${ }^{19}$ On the other hand, the tilt angle and elastic tilt modulus of lipid hydrophobic chains sensitively reflects the leaflet asymmetry. There is an inverse relationship between these two properties according to Eq. (2). The $K_{t}$ of the inner and outer leaflets in the asymmetric bilayer is $19.58 \pm 0.15$ and $22.29 \pm 0.15 \mathrm{mN} / \mathrm{m}$, respectively, at the early-log stage and $17.86 \pm 0.24$ and $20.67 \pm 0.33 \mathrm{mN} / \mathrm{m}$ at the stationary stage. The smaller $K_{t}$ at the inner leaflets was due to larger tilt angle of individual lipids (Table S2) at the inner leaflets Comparison with the symmetric bilayers, the $K_{t}$ of each leaflet in asymmetric membrane is smaller, except for the outer leaflet at the stationary stage. Also, the leaflet with a larger SA/lipid has a larger tilt angle (or a smaller tilt modulus), indicating that the asymmetry membrane alters their properties from the symmetric membrane and involves a lipid tilt and a lateral extension, referring a homogeneous deformation proposed by May et al. ${ }^{39}$ 
The electron density profiles (EDPs) along the z-axis of a membrane (the normal direction of the membrane) is utilized to evaluate the distribution of subgroups and overall atoms of bilayers. Figure $2 \mathrm{~A}$ shows overall EDPs having the same general symmetric shape and very similar in amplitude for the two asymmetric models. All demonstrated EDPs have shifted their leaflet boundary to $Z=0$. (i.e., the minimum point of the total EDP set to $Z=0$ ). Examining the component EDPs, only ethanolamine (Figure $2 B$ ), glycerol (Figure 2C), double bond, and cyclopropane groups (Figure 2D) reflect the asymmetric composition distribution between two leaflets in bilayer models (the inner leaflet is presented at the $z<$ 0 ; the outer leaflet is presented at the $z>0$ in the figures). The ethanolamine EDPs illustrated the asymmetric distribution of PE lipids, which is higher concentration in the outer leaflet in both models; and the glycerol EDPs illustrated the asymmetric distribution of PG lipids, which is a higher concentration in the inner leaflet in both models. The $\mathrm{CH}$ group EDPs reflects the distribution of the unsaturated fatty chains and the tails with a cyclopropane moiety. The CH EDPs at the early-log stage is symmetric due to a very close percentage of unsaturated tails (double bond), 53.8\% and 53.9\% in the outer and inner leaflets, respectively; while at the stationary membrane, the slightly higher percentage of unsaturated tails (29.6\%) in the inner leaflet, resulting in a higher intensity of the peak in the inner leaflet, compared to the less unsaturated tail (25.6\%) in the outer leaflet. Asymmetric peaks of cyclopropane EDPs are also observed in the stationary membranes, associating with the unequal distribution of cyclopropane which is $27.6 \%$ and $23.7 \%$ in the outer and inner leaflets, respectively. Non-zero electron density of double and cyclopropane was observed at the center of the membrane in both models, and this was quantified by the chain interdigitation of two leaflets (Table S3). The result of overall interdigitation indicates a deep penetration of lipids across the center of the bilayer at the similar level for both models. Asymmetric EDPs are not observed in all symmetric E. Coli IM models. 
$\mathbf{A}$

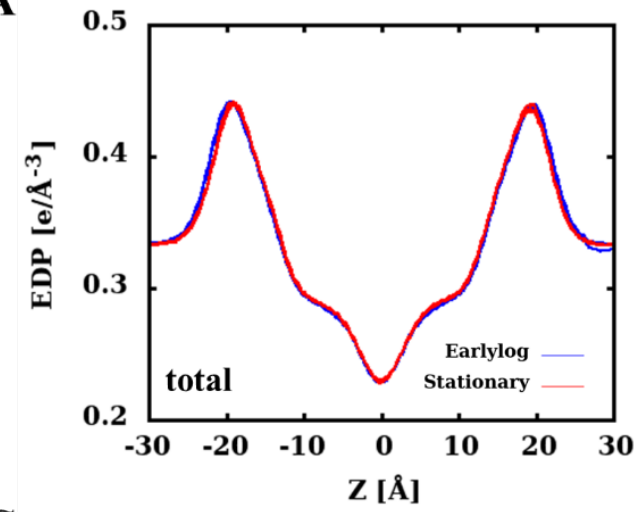

C

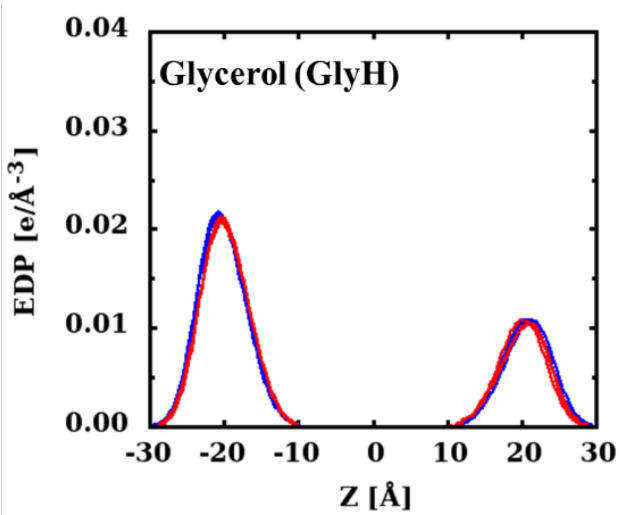

B

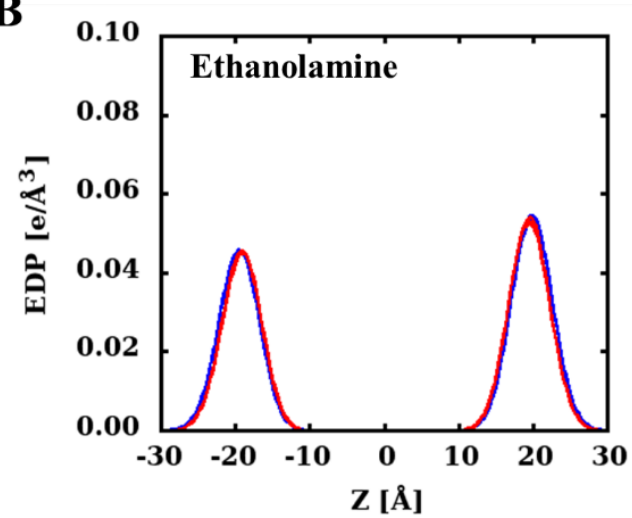

D

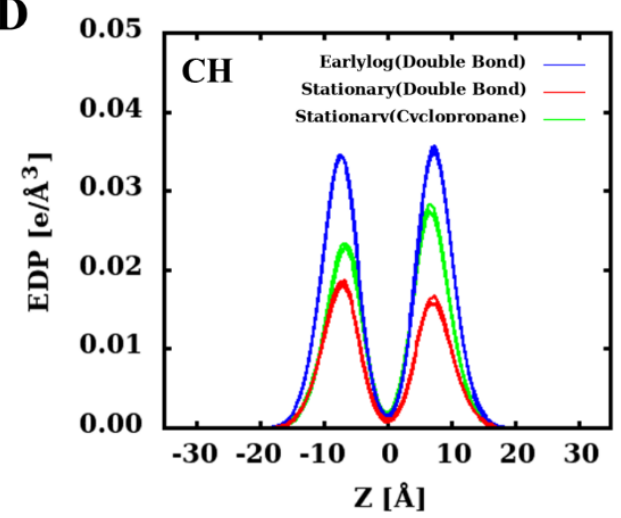

Figure 2. The electron density profiles of $(A)$ total atoms $(B)$ ethanolamine group (referring to PE lipids)

(C) Glycerol group (referring to PG lipids) and (D) CH group (referring double bond or cyclopropane moieties).

EDPs could be used to evaluate the thickness of the leaflets and the membranes. Table 4 shows the leaflet thicknesses of asymmetric bilayers, and thicknesses of symmetric bilayers for comparison. The $D_{c}$ of the inner and outer leaflets in the asymmetric bilayer is $14.34 \pm 0.02$ and $14.62 \pm 0.03 \AA$, respectively, at the early-log stage and $14.16 \pm 0.04$ and $14.50 \pm 0.01 \AA$ at the stationary stage. The $D_{B}$ of the inner and outer leaflets in the asymmetric bilayer is $18.32 \pm 0.02$ and $18.73 \pm 0.03 \AA$, respectively, at the early-log stage and $17.99 \pm 0.05$ and $18.36 \pm 0.04 \AA$ at the stationary stage. The $D_{H H}$ of the inner and outer leaflets in the asymmetric bilayer is $19.50 \pm 0.02$ and $19.56 \pm 0.22 \AA$, respectively, at the early-log stage and $19.17 \pm 0.09$ and $19.37 \pm 0.08 \AA$ at the stationary stage. The $D_{B}$ and $D_{C}$ in the inner leaflet are significantly thinner, compared to the outer leaflets in individual stage of membrane, which is consistent with the result of SA/lipid in leaflets. Comparison between symmetric and asymmetric models, at the stationary stage, smaller $D_{C}$ and $D_{B}$ of the asymmetric inner leaflet model are found, while larger $D_{C}$ and $D_{B}$ of symmetric outer leaflet model are found. At the early-log stage, only a difference in $D_{B}$ is significant. Both inner and outer asymmetric leaflets have a lower $D_{B}$ compared with the corresponding symmetric models. A 
observed correlation that the leaflet thickness decreases with a increasing tilt angle, consistently following the homogeneous deformation theory. ${ }^{39}$

Table 4. Thickness of model membranes.

\begin{tabular}{cccc}
\hline \hline Model & $D_{C}\left(\AA^{2}\right)$ & $D_{B}\left(\AA^{2}\right)$ & $D_{H H}\left(\AA^{2}\right)$ \\
\hline earlylog membrane & $28.96 \pm 0.04$ & $37.04 \pm 0.05$ & $39.07 \pm 0.22$ \\
outer leaflet & $14.62 \pm 0.03$ & $18.73 \pm 0.03$ & $19.56 \pm 0.22$ \\
inner leaflet & $14.34 \pm 0.02$ & $18.32 \pm 0.02$ & $19.50 \pm 0.02$ \\
stationary membrane & $28.66 \pm 0.04$ & $36.35 \pm 0.09$ & $38.53 \pm 0.16$ \\
outer leaflet & $14.50 \pm 0.01$ & $18.36 \pm 0.04$ & $19.37 \pm 0.08$ \\
inner leaflet & $14.16 \pm 0.04$ & $17.99 \pm 0.05$ & $19.17 \pm 0.09$ \\
pre-equilibrium membranes & & & \\
earlylog-outer & $29.32 \pm 0.10$ & $38.62 \pm 0.16$ & $39.33 \pm 0.08$ \\
(leaflet) & $(14.66 \pm 0.03)$ & $(19.31 \pm 0.05)$ & $(19.67 \pm 0.06)$ \\
earlylog-inner & $28.86 \pm 0.10$ & $38.08 \pm 0.15$ & $39.27 \pm 0.29$ \\
(leaflet) & $(14.43 \pm 0.03)$ & $(19.04 \pm 0.05)$ & $(19.63 \pm 0.15)$ \\
stationary-outer & $28.65 \pm 0.22$ & $36.30 \pm 0.13$ & $38.20 \pm 0.28$ \\
(leaflet) & $(14.32 \pm 0.06)$ & $(18.15 \pm 0.05)$ & $(19.10 \pm 0.13)$ \\
stationary-inner & $28.62 \pm 0.10$ & $36.31 \pm 0.06$ & $38.47 \pm 0.22$ \\
(leaflet) & $(14.31 \pm 0.03)$ & $(18.16 \pm 0.02)$ & $(19.23 \pm 0.08)$ \\
\hline \hline
\end{tabular}

Table 4 also shows that the overall bilayer thicknesses (combinations of the two leaflets). The thicknesses of early-log membrane are slightly thicker than the stationary membranes, which is consistent with a smaller SA/lipid of early-log membrane. Specifically, the hydrophobic core thickness $(2 D c)$ is $29.96 \pm 0.04$ $\AA$ and $28.66 \pm 0.04 \AA$ at the early-log and the stationary membranes, respectively. Comparison with other symmetric $E$. Coli inner membrane models, the hydrophobic thicknesses of $E$. Coli cytoplasmic membrane in the bacterial lifecycle models ${ }^{19}$ were 30.0 to $30.6 \AA$. The hydrophobic thickness of another bacterial cytoplasmatic membrane model, the Top $6,{ }^{18}$ was $29.8 \pm 0.1 \AA$. Although there is a slight variation between these models, they are statistically identical to $29.4 \pm 1 \AA$ from the previous survey of the hydrophobic thickness of E. Coli cytosolic membrane proteins through the Orientations of Proteins in Membranes (OMP) database. The bilayer thicknesses $\left(D_{B}\right)$ are $37.04 \pm 0.05 \AA$ and $36.35 \pm 0.09 \AA$ at the early-log and stationary membranes, respectively. The head-to-head distances $\left(D_{H H}\right)$ are $39.07 \pm 0.22 \AA$ and $38.53 \pm 0.16 \AA$ at the early-log and the stationary membranes, respectively. Significant difference between the two stages in $D_{B}$ and $2 D_{C}$, but not in $D_{H H}$ (Table S1) are observed. $D_{H H}$ is less sensitive to the bacterial development stages 
in model membranes due to the same head group composition in the model membranes at different stages of the bacterial life cycle.

Figure 3 and S5 show the pressure profiles along the z-axis of a membrane (the normal direction of the membrane) which were utilized to evaluate the lateral surface tension and spontaneous bending (curvature) of bilayer. The pressure profiles show a significant oscillation at the water-bilayer interfaces, driven by nonbonding interactions (the electrostatic and van der Waals interactions) of the lipid headgroups at the surface of bilayer. The pressure decays to zero away from the water-lipid interface toward to the water box. Within the hydrophobic core, the profile remains at high pressure and the oscillations are contributed by the lipid tails. For instance, the midplane peak associates with the interactions between the end of lipid tails. Also, there are more clear peaks next to the midplane in both leaflets of the stationary bilayer, compared to the early-log bilayer. The position of the peaks is right at the maximum intensity of the cyclic EDP (Figure 2D), indicating these peaks associates with the cyclopropane moiety. The surface tension of each leaflet (Table 5) was computed by integration of the pressure profile along the z-axis, resulting in the same absolute value with opposite signs for two leaflets in individual bilayers and leading to a zero surface tension of each bilayer which matches the simulation setting of tensionless condition. Although the absolute values of monolayer surface tension $(\gamma)$ of all modeled membranes were varied, the outer model of symmetric stationary membrane was significantly lower compared to others and other membranes have no statistically significant difference.

Table 5 also lists the first derivative of bending free energy per lipid at the zero curvature, $F^{\prime}(0)$, indicating the bending tendency of individual monolayers. A minus sign was add to the $F^{\prime}(0)$ value of inner leaflet so that the lipid direction in the monolayer was inversed as the same direction as the outer leaflet (the headgroups are in the $z$ position direction and the tails are in the $z$ negative direction). Therefore, the $F^{\prime}(0)$ for all monolayers could be directly used for comparison and included in statistics without affecting by the direction of lipid aligning on the $z$ axis. Note that if the pressure profile is perfectly symmetrical between two leaflets, $F^{\prime}(0)$ of the leaflets is exactly same value. The $F^{\prime}(0)$ at the inner and outer leaflets in the asymmetric early-log membranes was $-0.0406 \pm 0.0053 \mathrm{kcal} / \mathrm{mol} / \AA ̊$ and $-0.0398 \pm 0.0167 \mathrm{kcal} / \mathrm{mol} / \AA$, respectively, having a highly similarity in both leaflets with positive spontaneous curvatures; on the other hand, the $F^{\prime}(0)$ in the asymmetric stationary membrane is opposite between leaflets, specifically this was $0.0090 \pm 0.0059 \mathrm{kcal} / \mathrm{mol} / \AA ̊ \AA$ in the inner leaflet (a negative spontaneous curvature) and $-0.0513 \pm 0.0226$ $\mathrm{kcal} / \mathrm{mol} / \AA$ in the outer leaflet (a positive spontaneous curvature). In the symmetric membranes, the negative $F^{\prime}(0)$ of the inner models, leading to a positive spontaneous curvature, was significant different 
from that of the outer models, having an opposite spontaneous curvature in both stages. The $F^{\prime}(0)$ of a whole bilayer (Table S4; the integral range is from the bottom to the top water-lipid interfaces) for the asymmetric early-log and stationary models was $0.0008 \pm 0.0118 \mathrm{kcal} / \mathrm{mol} / \AA ̊$ (a nearly zero spontaneous curvature) and $-0.603 \pm 0.0167 \mathrm{kcal} / \mathrm{mol} / \AA$ (a positive curvature), respectively, indicating that the stationary membrane likely tends to bend toward a positive curvature but the early-log membrane unlikely tend to bend due to a flat curvature. In the symmetric membranes, whole bilayer $\mathrm{F}^{\prime}(0)$ has no significant different among all models though the inner models have a positive spontaneous curvature while the outer models have a negative spontaneous curvature. Intuitively, the curvature is induced by molecular shape of monolayer-forming lipid, i.e., a larger headgroup with a shorter tail induces a more negative curvature, which has been observed in both modeling and experimental works, ${ }^{41,44-46}$ focusing on single-component bilayers. For instance, Venable et al. ${ }^{41}$ reported that the $\mathrm{F}^{\prime}(0)$ of PE lipid monolayer (POPE bilayer; $0.2041 \pm 0.0031 \mathrm{kcal} / \mathrm{mol} / \AA ̊ \AA)$ ) is larger than that of POPG lipid monolayer $(0.0036 \pm 0.0024$ $\mathrm{kcal} / \mathrm{mol} / \AA ̊ \AA)$. However, the curvature of multi-component lipid monolayers is still very unclear though it has been estimated by ideal mixing (linear combination in 2 or 3 lipids with sterol). ${ }^{44}$ The molecular interaction of lipids such as the electrostatic interaction and hydrogen bonding strongly affects the monolayer curvature resulting in challenge of predicting the curvature of multi-component lipid monolayers (and bilayers).

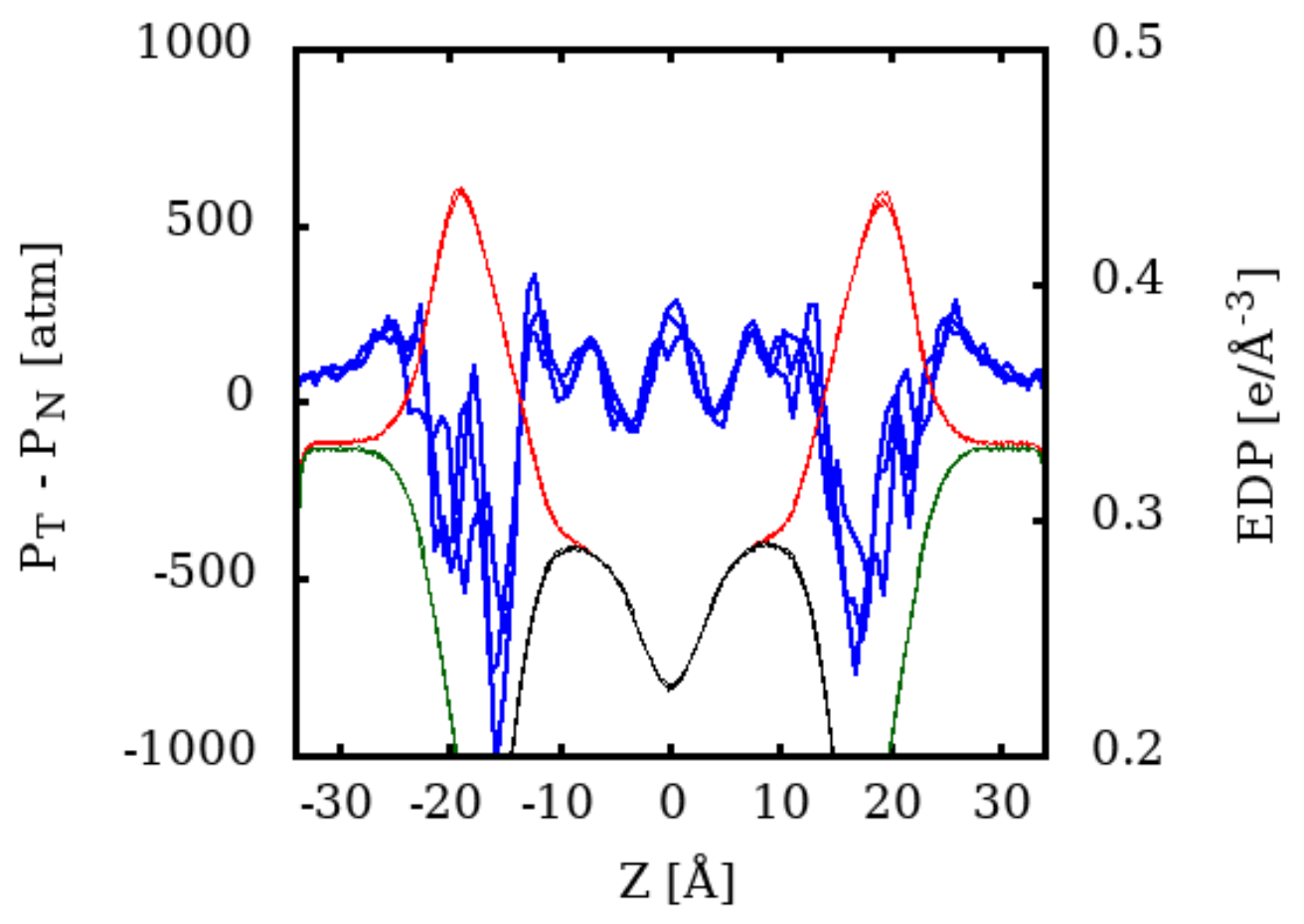


Figure 3. The pressure profiles (blue) of stationary models. The total (red), water (green) and hydrophobic core (black) EDP profiles are also shown as references.

Table 5. Surface tension and the first derivative bending free energy (spontaneous curvature).

\begin{tabular}{ccc}
\hline \hline Model & $\gamma($ dyn/cm $)$ & $F^{\prime}(0)(\mathrm{kcal} / \mathrm{mol} / \mathrm{A})$ \\
\hline $\begin{array}{c}\text { earlylog membrane } \\
\text { outer leaflet } \\
\text { inner leaflet }\end{array}$ & $1.005 \pm 0.1818$ & $-0.0398 \pm 0.0167$ \\
stationary membrane & $1.005 \pm 0.1818$ & $-0.0406 \pm 0.0053$ \\
outer leaflet & & \\
inner leaflet & $2.617 \pm 0.777$ & $-0.0513 \pm 0.0226$ \\
symmetric membranes & $2.617 \pm 0.777$ & $0.0090 \pm 0.0059$ \\
early-log-outer & $1.401 \pm 0.285$ & \\
early-log-inner & $2.003 \pm 0.988$ & $-0.0718 \pm 0.0256$ \\
stationary-outer & $0.620 \pm 0.280$ & $0.0122 \pm 0.0184$ \\
stationary-inner & $1.939 \pm 0.382$ & $-0.0667 \pm 0.0222$ \\
\hline \hline
\end{tabular}

The deuterium order parameter $\left(S_{C D}\right)$ of the acyl chain in all model membranes follows the general trend, decreasing at the location of a double bond and a cyclopropane ring known to induce local disordering of the chain packing in the bilayer. The $S_{C D}$ also drops deeper at the end of the chain due to the free rotation of the methyl group. All saturated acyl acid tails (sn-1) and unsaturated acyl acid tails (sn-2) demonstrated that the stationary stage is more disordered compared to the early-log stage in all range of tails, good agreement with our previous study. ${ }^{19}$ Figures $4 \mathrm{~A}-\mathrm{B}$, and S2-S4 show the individual lipid chain $S_{C D}$ in each leaflet affecting by asymmetric lipid component distribution. The significant differences between two leaflets of individual lipid $S_{C D}$ are found.

Table 6. The sum of C4 to C14 Scd sn-1 and sn-2 chains of model membranes

\begin{tabular}{ccc}
\hline \hline Model & $s n-1$ & $s n-2$ \\
\hline earlylog membrane & $0.192 \pm 0.001$ & $0.148 \pm 0.001$ \\
outer leaflet & $0.198 \pm 0.001$ & $0.153 \pm 0.001$ \\
inner leaflet & $0.187 \pm 0.001$ & $0.143 \pm 0.0001$ \\
stationary membrane & $0.184 \pm 0.001$ & $0.145 \pm 0.001$ \\
outer leaflet & $0.193 \pm 0.001$ & $0.151 \pm 0.001$ \\
inner leaflet & $0.177 \pm 0.001$ & $0.139 \pm 0.001$ \\
symmetric membranes & & \\
early-log-outer & $0.203 \pm 0.001$ & $0.153 \pm 0.0003$ \\
early-log-inner & $0.197 \pm 0.002$ & $0.149 \pm 0.001$ \\
stationary-outer & $0.189 \pm 0.001$ & $0.147 \pm 0.001$
\end{tabular}


A

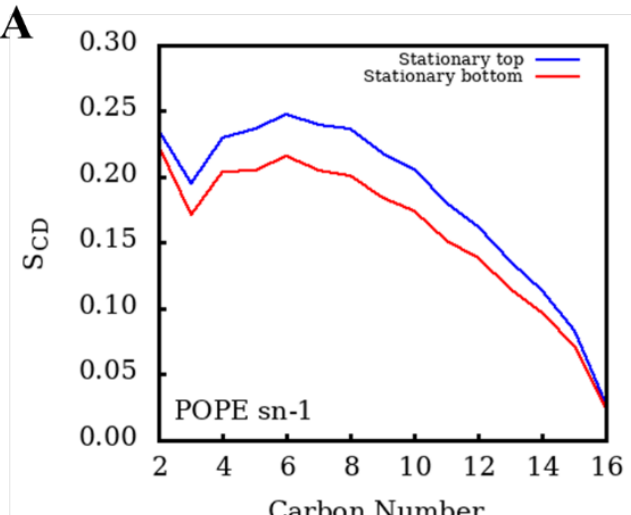

C

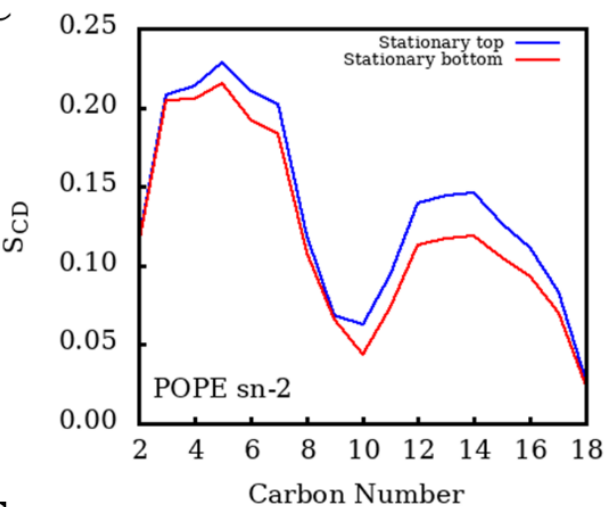

$\mathbf{E}$

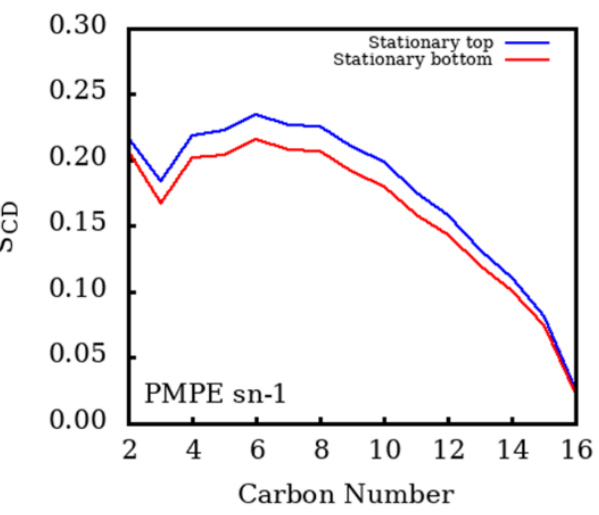

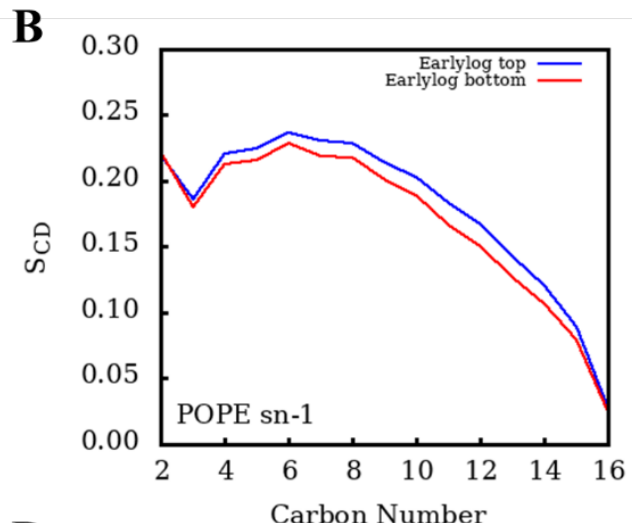

D

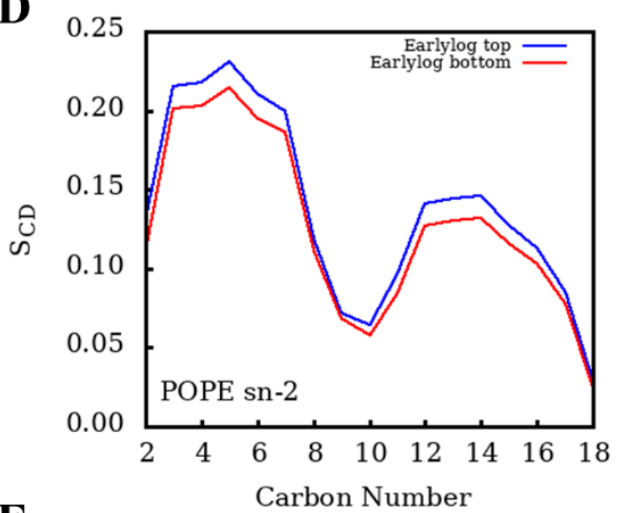

$\mathbf{F}$

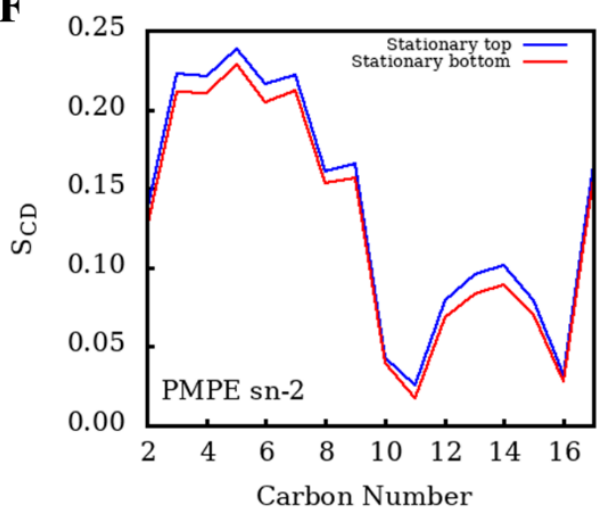

Figure 4. The $S_{C D}$ profiles of POPE sn-1 chain at (A) the stationary and (B) the earlogy stages, respectively; and POPE sn-2 chain at (C) the stationary and (D) the earlogy stages; and PMPE (E) sn-1 chain and (F) sn-2 chain at the stationary stage. The outer leaflets consistently have a higher order parameter in whole range of carbons in all presenting lipids. 
Since the $S_{C D}$ of the monounsaturated $s n-1$ tail has a similar order profile, the average $S_{C D}$ of $C 4$ to C14 of lipids over each bilayer leaflet and bilayer (Table 6) could evaluate the deviation of the disordering between bacterial life cycle stages. For comparison, the same calculation for $s n-2 S_{C D}$ is listed as well. The average $S_{C D}$ shows lipids in the inner leaflet are more disordered compared to the outer leaflet in both stages. In the asymmetric bilayer, the average $S_{C D}$ of $s n-1$ of the inner and outer leaflets is $0.187 \pm 0.001$ and $0.198 \pm 0.001$, respectively, at the early-log stage and $0.177 \pm 0.001$ and $0.193 \pm 0.001$ at the stationary stage. The average $S_{C D}$ of $s n-2$ of the inner and outer leaflets is $0.143 \pm 0.0001$ and $0.153 \pm 0.001$, respectively, at the early-log stage and $0.139 \pm 0.001$ and $0.151 \pm 0.001$ at the stationary stage. The $S_{C D}$ reflects on leaflet asymmetry and the values are significantly different between two leaflets at the same E. Coli developmental stage. Comparison with the symmetric bilayers, the $S_{C D}$ of $s n-1$ of each leaflet model is significant lower in asymmetric membrane, except for the outer leaflet models at the stationary stage having no significant difference. For the $s n-2$ tail, on the other hand, the $S_{C D}$ of inner leaflet models is significant lower in asymmetric membrane at both stages while the $S_{C D}$ of outer leaflet model is significant higher in asymmetric membrane at stationary stage and no significant difference of the $S_{C D}$ of outer leaflet model at the early-log stage. This result corresponds to the SA/lipid which excellently agrees with a known relationship that the $S_{C D}$ is inversely associated with the surface area in general. ${ }^{47}$

Figure 4C-D, S3, and Table S5 shows the average result of regional $S_{C D}$ over all lipid sn-2 chains with a double bond. Figure $4 \mathrm{E}-\mathrm{F}, \mathrm{S} 4$, and Table $\mathrm{S} 6$ shows the average $S_{C D}$ of lipids $s n-2$ chain with a cyclopropane at the regional carbon positions. The $S_{C D}$ of $s n-2$ chain results in agreeing with the report from Pandit and Klauda ${ }^{18}$ and the experiment. ${ }^{48}$ Comparison across leaflets in the same asymmetric membrane, the average $S_{C D}$ of outer leaflet for individual regions is significantly higher at both stages, instead of the double bond location ( $\mathrm{C9}$ and $\mathrm{C} 10$ ) and the cyclopropane region (C8-C10) having no significant difference, indicating the order parameter is restricted by the molecular structure and less affected by lipid packing. Table S5-6 show the $S_{C D}$ of symmetric bilayers have a similar tendency as well. Comparison between symmetric and asymmetric models, the consistent lower $S_{C D}$ of asymmetric inner leaflet models is found at non-double bond positions at the early-log stage and at C3-C7 at stationary stage. The significant lower order parameters at C3-C8 (Table S5) were seen at the stationary stage for the double bond sn-2 chain in both leaflets and the bilayer, indicating the effect of the cyclopropane moiety from other types of lipids (PMPE, PMPG, and QMPE) in decreasing the order of acyl fatty chain at the stationary stage. 
Two-dimensional radial distribution functions (2D-RDFs) of phosphate atoms in the individual leaflets and a whole bilayer are generated between homo- and hetero-lipid pairs PE-PE, PG-PG, PE-PG, and lipid-lipid of two models. Figure 5A demonstrates 2D-RDFs for a whole bilayer of $E$. coli in the stationary stage. The headgroup-headgroup pair is calculated for all, i.e., without distinguishing the lipid chain types. The 2D RDFs show strong two peaks for PE-PE, PE-PG, but not for the PG-PG pair. The low intensity of PG-PG 2DRDFs is due to the repulsion between PG headgroups. The PG-PG 2D-RDFs show variation among three replicas, especially in low PG concentrations (i.e., outer leaflets). To capture the average 2D RDF profile, the last 200 ns data after equilibrium (i.e., 200-400ns) was taken to generate 2D RDF profile for analysis. The two peaks of each 2D RDF profile have been fitted with Gaussian function and the resulting peak positions (Table S7) increase in the order of PE-PE, PE-PG, and PG-PG pairs. The result highly correlates to the formation of hydrogen bonds in the lipid pairs (Figure 5B-C). PE lipids have a stronger self-association since PE lipids can be either a donor or acceptor to form intra-lipid hydrogen bonds. In PE-PG pair, amines in PE act as donors, and hydroxyl groups in PG act as acceptors to form inter-lipid hydrogen bonds. A dual peak on the profile is due to the two potential conformations of lipids in their pairs.

Gaussian function fits show the positions of two major peaks of 2D RDF at the stationary stage tend to be slightly larger compared to the early log stage. A slightly larger position of the first peak in the lipid-lipid 2D RDF of both leaflets and the bilayer at the stationary stage compared to the early log stage. The first peak of lipid-lipid 2D RDF in the stationary and early-log bilayers is $6.15 \pm 0.01 \AA$ and $6.12 \pm 0.01 \AA$, respectively, caused by mainly PE lipid pair in model membranes. The first peak of PE-PE 2D RDF in the stationary and early-log bilayers is $6.07 \pm 0.01 \AA$ and $6.03 \pm 0.01 \AA$, respectively. A positive correlation between the first peak position of the lipid-lipid 2D RDF and the SA/lipid has been observed; the position of two peaks in PE-PE and PE-PG 2D RDF profiles, which has been reported to be close to the projection distance between paired phosphates on the $x-y$ plane. ${ }^{17}$ Thus, this correlation is due to a high content of PE-PE pair in the model membranes. There is no significant difference in 2D-RDF peak positions between symmetric and asymmetric models. 


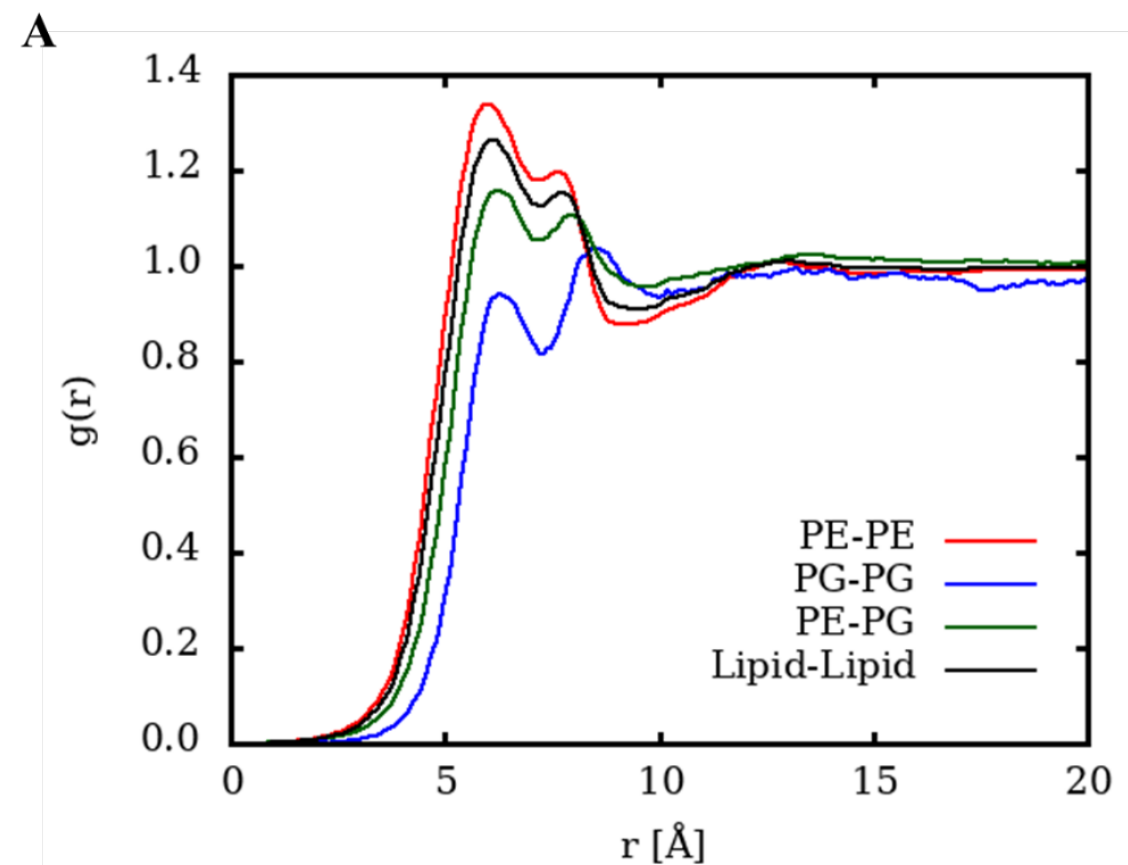

B

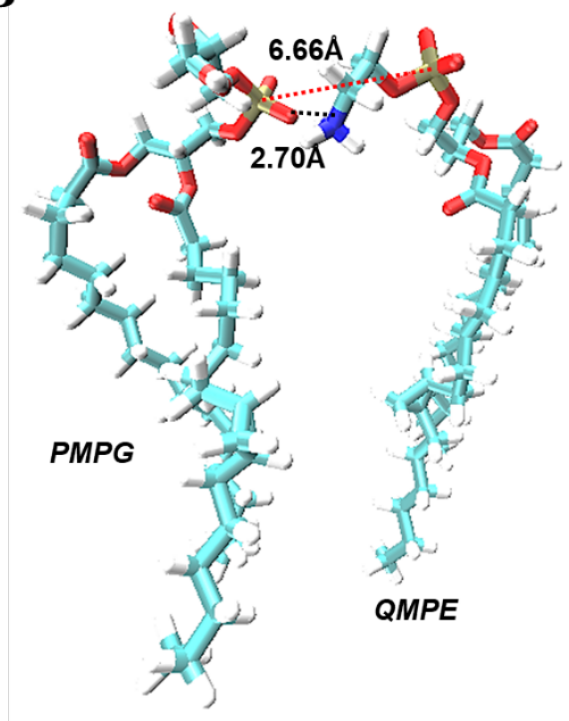

C

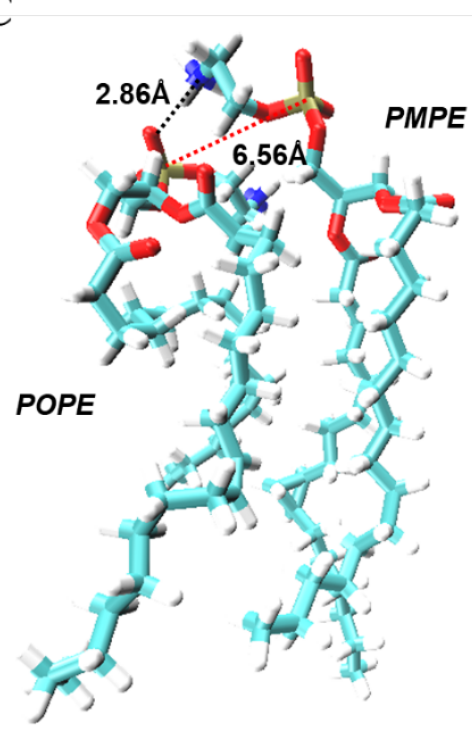

Figure 5. (A) 2D radial distribution functions of phosphate atoms for PE-PE (in red), PE-PG (in blue), PE-PG (in green) and lipid-lipid (in black, all lipid pairs) pairs. Snapshots of (B) POPE-PMPE pair and (C) PMPGQMPE pair showing hydrogen bonding (black) between amine group and PO and the phosphatephosphate distance (red). The 2D-RDF is calculated using data of whole membrane from 200 to $400 \mathrm{~ns}$ and the snapshots are taken from the outer leaflet at 400 ns of the E. coli stationary phase (replica 3 ).

\section{Discussion and Conclusion}


This work is the first to model the asymmetric lipid composition between two leaflets in the inner membrane of E. Coli through MD simulation using CHARMM36 lipid force field. The models of early-log and stationary membranes are used to study two distinct regimes, growth stage and plateau stage, during the bacterium life cycle. The major distinction between these two models is that the stationary membrane contains lipids with a cyclopropane moiety in the sn-2 chain, and assuming these bacterial species lipids are not presenting in the early-log membrane. For leaflet asymmetry, the lipid composition was guided to match the "positive inside" rule that more positive residues of transmembrane protein reside at the inner leaflet of the membrane led to more lipids with negative headgroups to neutralize the membrane. Therefore, a lower PE/PG ratio (2.8) was set in the inner leaflet, while a higher PE/PG ratio (6.8) was set in the outer leaflet. The overall PE:PG ratio was set to 4.1. The result revealed variations of properties in bulk membranes or in individual lipids at the atomic scale for investigating how lipid diversity and asymmetric lipid concentrations affect these properties. The significant variances between the two bacterial developmental stages are that the SA/lip is larger, the thickness $\left(2 D_{C}\right.$ and $\left.D_{B}\right)$ is thinner, the tilt modulus is smaller, and the $S_{C D}$ of $s n-1$ and $s n-2$ tails are lower for membranes at the stationary stage. Also, a positive spontaneous curvature is found at the stationary stage. The result is like the previous publications ${ }^{18-19}$ probing in lipid diversity on E. Coli cell symmetric membrane.

A

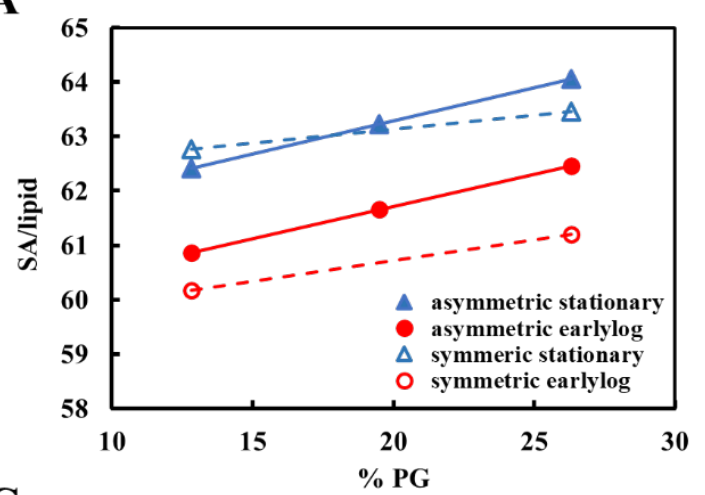

C

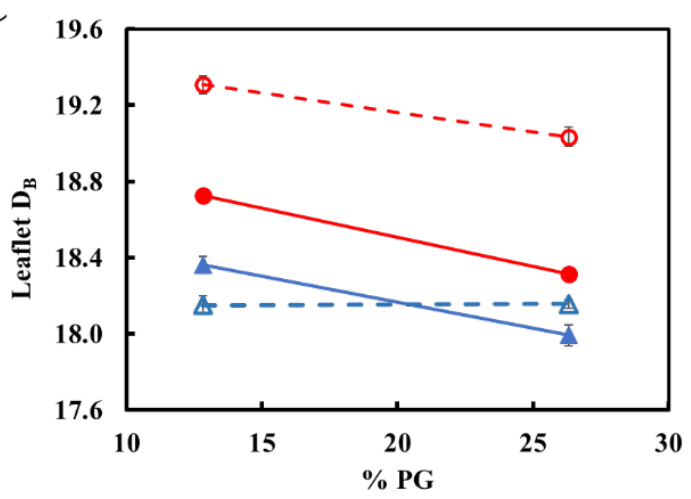

B

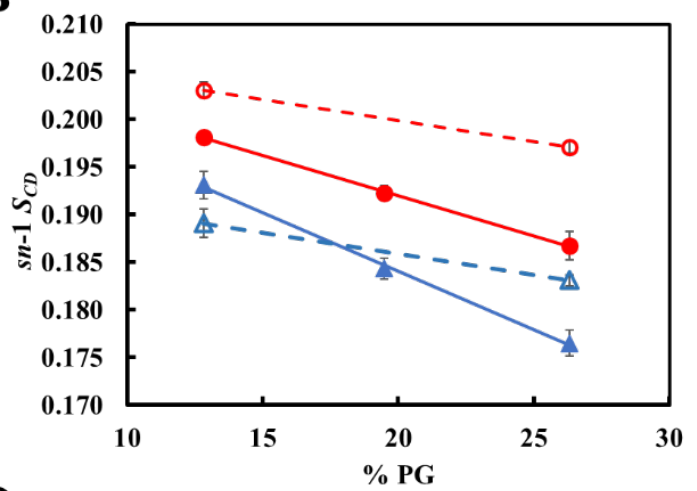

D

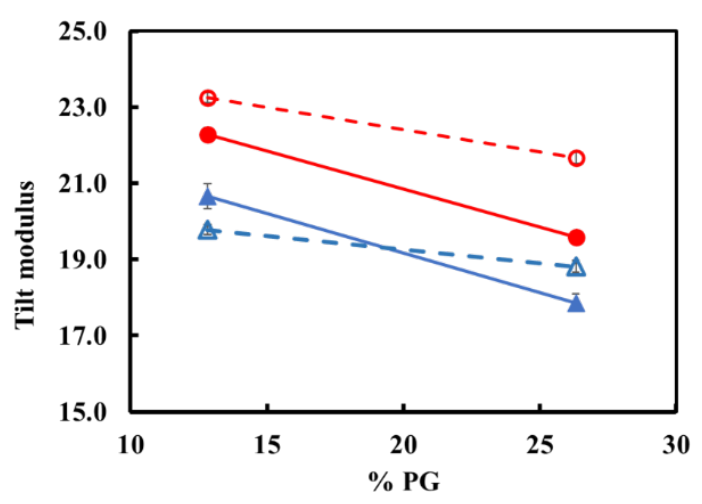


Figure 6. The (A) SA/lipid, (B) average of sn-1 $S_{C D}(C)$ the leaflet thickness, and (D) the tilt modulus of individual leaflets at the two stages. The $12.8 \%$ and $26.3 \%$ of PG represent the outer and inner leaflets, respectively, of both membrane models, and $19.5 \%$ of PG represents the average of whole membrane. Assuming a linear correlation between membrane properties and \% PG, the tendency lines are showed in the figures.

Lipid composition asymmetry, in our simulation, is described by the unequal amount of PG lipids in the lipid leaflets that $12.8 \%$ of PG in the outer, $26.3 \%$ of PG in inner leaflets, and $19.5 \%$ in a whole asymmetric membrane. The SA/lipid (Figure 6A), the average $S_{C D}$ of $s n$-1 saturated tails (C4-C14) (Figure 6B), the leaflet thickness $D_{B}$ (Figure $6 \mathrm{C}$ ), and the tilt modulus $K_{t}$ have been demonstrated as a function of \% PG, showing that the surface area per lipid increase with an increasing \%PG while the deuterium order parameter, the leaflet thickness, and the tilt modulus decreases with an increasing \%PG in both symmetric and asymmetric models. Again, these properties vary slightly more between symmetric and asymmetric membranes at the early-log stage with the same \%PG (the same leaflets), compared to the stationary stage (see details in Result section). Furthermore, the properties in the asymmetric membranes are more dependent on \%PG compared to that in the symmetric membranes, i.e., a greater absolute value of the slope in the Figure 6. Unbalanced lipid composition in two lateral monolayers of our asymmetric membranes effect on leaflet membrane properties. In the geometric view, the total surface area in the inner and outer leaflets is equal; so that, the inner leaflet (less lipids and high \%PG) has a bigger surface area per lipid, which requires a more lipid tilting (a bigger tilt angle) and results a smaller tilt modulus. At the same time, the total volume of lipids in constant (the incompressibility assumption) also leads that the inner leaflet has a small packing volume, resulting a small leaflet thickness. The above demonstrates the self-adjustment of lipid monolayer affecting bulky properties of membrane. A stronger selfadjustment in membrane/lipid properties at the asymmetric stationary membrane leads to a less change of properties from the symmetric models. A varied derivative of bending free energy (spontaneous curvature) between the outer and inner leaflets at the stationary bilayer were found, compared with the less sensitive in the early-log bilayer, that the stationary bilayer tends to a positive spontaneous curvature and the early-log bilayer tends to be a flat spontaneous curvature. In symmetric membranes, the properties have similar relationships, corresponding to the surface area per lipid, but no asymmetric effect and same tilt in lateral leaflets. The individual lipids and their interactions also affect the leaflet properties. The inner leaflets of the stationary stage have a bigger surface area per lipid due to containing more PG 
lipid and lipid tail with a cyclopropane while the outer leaflets of early-log have a smaller SA/lipid due to less PG lipid and no tail with a cyclopropane. The surface area per lipid and other properties show less difference between asymmetric and symmetric membranes at the stationary stage. One explanation is that the lipid tail containing a cyclopropane have a more space of freedom which could make a significant adjustment to reduce the effect of lipid composition asymmetry; on the other hand, the early-log membranes without the cyclic tails must vary more to match the effect of lipid composition asymmetry.

Overall, our pioneer study on the leaflet asymmetry in lipid composition and variations in the colony life cycle states of the $E$. coli cytoplasmic membrane demonstrates a significant difference between two leaflets in their membrane properties, including the SA/lipid, thickness, tilt modulus, and order parameters. Similar to the previous symmetric model with lipid diversity, our model also successfully describes significant changes of a selected set of whole membrane properties (SA/lipid, thickness, and order parameter) in different stages. Our MD simulation support that the individual leaflet with different lipid composition resulting in a difference of its properties, i.e., lipid packing in this work, led to a significant change of whole membrane properties. This work provides an initial assessment of the effect of asymmetric lipid distribution, but how this leaflet asymmetry affects the membrane properties and integrity of the bilayer remains unclear. The lipid composition asymmetry and lipid diversity with an appropriate mixture of various lipids to model organism and organelle membranes provides a more accurate representation of the membrane properties and significantly differ from the two-lipid models in the earlier simulations. We believe that the asymmetric cytoplasmic membrane model reflects a more realistic membrane that will allow for improved studies of interactions, structure and dynamics of membrane-associated molecules.

\section{Supporting Information}

Additional figures and tables on membrane properties (t-test for membrane properties between two stages and two leaflets, the tilt angles of individual lipids, the lipid interdigitations, the $S_{C D}$ of leaflets and membranes, the 2D-RDF of leaflets and membranes, time functions of leaflet SA/lipid), the pressure profiles as well as simulation movie files, are presented in the supplementary materials section.

\section{Acknowledgements}


The high-performance computational resources used for this research are Deepthought2, Bluecrab, and Bridges-2 (the early user program/XSEDE) maintained by the Division of Information Technology at the University of Maryland, the Maryland Advanced Research Computing Center (MARCC), and the Pittsburgh Supercomputing Center (PSC), respectively. This research was supported in part by NSF (CHE-2003912 and CHE-2029900).

\section{References}

1. Walther, T. C.; Farese, R. V., Lipid droplets and cellular lipid metabolism. Annu Rev Biochem 2012, 81, 687-714.

2. Wymann, M. P.; Schneiter, R., Lipid signalling in disease. Nat Rev Mol Cell Biol 2008, 9 (2), 16276.

3. Casares, D.; Escribá, P. V.; Rosselló, C. A., Membrane Lipid Composition: Effect on Membrane and Organelle Structure, Function and Compartmentalization and Therapeutic Avenues. Int J Mol Sci 2019, 20 (9).

4. van Meer, G.; Voelker, D. R.; Feigenson, G. W., Membrane lipids: where they are and how they behave. Nat Rev Mol Cell Biol 2008, 9 (2), 112-24.

5. Zwaal, R. F.; Bevers, E. M.; Comfurius, P.; Rosing, J.; Tilly, R. H.; Verhallen, P. F., Loss of membrane phospholipid asymmetry during activation of blood platelets and sickled red cells; mechanisms and physiological significance. Mol Cell Biochem 1989, 91 (1-2), 23-31.

6. Verkleij, A. J.; Zwaal, R. F.; Roelofsen, B.; Comfurius, P.; Kastelijn, D.; van Deenen, L. L., The asymmetric distribution of phospholipids in the human red cell membrane. A combined study using phospholipases and freeze-etch electron microscopy. Biochimica et biophysica acta 1973, 323 (2), 17893.

7. Lorent, J. H.; Levental, K. R.; Ganesan, L.; Rivera-Longsworth, G.; Sezgin, E.; Doktorova, M.; Lyman, E.; Levental, I., Author Correction: Plasma membranes are asymmetric in lipid unsaturation, packing and protein shape. Nat Chem Biol 2020, 16 (6), 710.

8. Kumar, A.; Gupta, C. M., Red cell membrane abnormalities in chronic myeloid leukaemia. Nature 1983, 303 (5918), 632-3.

9. Nikaido, H., Molecular basis of bacterial outer membrane permeability revisited. Microbiol Mol Biol Rev 2003, 67 (4), 593-656.

10. Furse, S.; Scott, D. J., Three-Dimensional Distribution of Phospholipids in Gram Negative Bacteria. Biochemistry 2016, 55 (34), 4742-7.

11. Dufourc, E. J.; Smith, I. C. P.; Jarrell, H. C., The role of cyclopropane moieties in the lipid properties of biological membranes: a deuterium NMR structural and dynamical approach. Biochemistry 1984, 23 (10), 2300-2309.

12. Grogan, D. W.; Cronan, J. E., Cyclopropane ring formation in membrane lipids of bacteria. Microbiol Mol Biol Rev 1997, 61 (4), 429-41.

13. Klauda, J. B., Perspective: Computational modeling of accurate cellular membranes with molecular resolution. J Chem Phys 2018, 149 (22), 220901.

14. Leonard, A. N.; Wang, E.; Monje-Galvan, V.; Klauda, J. B., Developing and Testing of Lipid Force Fields with Applications to Modeling Cellular Membranes. Chem Rev 2019, 119 (9), 6227-6269.

15. Monje-Galvan, V.; Klauda, J. B., Modeling Yeast Organelle Membranes and How Lipid Diversity Influences Bilayer Properties. Biochemistry 2015, 54 (45), 6852-61. 
16. Jo, S.; Lim, J. B.; Klauda, J. B.; Im, W., CHARMM-GUI Membrane Builder for mixed bilayers and its application to yeast membranes. Biophys $J$ 2009, 97 (1), 50-8.

17. Yu, Y.; Klauda, J. B., Modeling Pseudomonas aeruginosa inner plasma membrane in planktonic and biofilm modes. J Chem Phys 2018, 149 (21), 215102.

18. Pandit, K. R.; Klauda, J. B., Membrane models of E. coli containing cyclic moieties in the aliphatic lipid chain. Biochimica et biophysica acta 2012, 1818 (5), 1205-10.

19. Khakbaz, P.; Klauda, J. B., Probing the importance of lipid diversity in cell membranes via molecular simulation. Chem Phys Lipids 2015, 192, 12-22.

20. Wu, E. L.; Fleming, P. J.; Yeom, M. S.; Widmalm, G.; Klauda, J. B.; Fleming, K. G.; Im, W., E. coli outer membrane and interactions with OmpLA. Biophys J 2014, 106 (11), 2493-502.

21. Matsuzaki, K.; Sugishita, K.; Harada, M.; Fujii, N.; Miyajima, K., Interactions of an antimicrobial peptide, magainin 2, with outer and inner membranes of Gram-negative bacteria. Biochimica et biophysica acta 1997, 1327 (1), 119-30.

22. Lohner, K.; Prenner, E. J., Differential scanning calorimetry and X-ray diffraction studies of the specificity of the interaction of antimicrobial peptides with membrane-mimetic systems. Biochimica et biophysica acta 1999, 1462 (1-2), 141-56.

23. Oursel, D.; Loutelier-Bourhis, C.; Orange, N.; Chevalier, S.; Norris, V.; Lange, C. M., Lipid composition of membranes of Escherichia coli by liquid chromatography/tandem mass spectrometry using negative electrospray ionization. Rapid Commun Mass Spectrom 2007, 21 (11), 1721-8.

24. Gidden, J.; Denson, J.; Liyanage, R.; Ivey, D. M.; Lay, J. O., Lipid Compositions in Escherichia coli and Bacillus subtilis During Growth as Determined by MALDI-TOF and TOF/TOF Mass Spectrometry. Int J Mass Spectrom 2009, 283 (1-3), 178-184.

25. Hughes, A. V.; Patel, D. S.; Widmalm, G.; Klauda, J. B.; Clifton, L. A.; Im, W., Physical Properties of Bacterial Outer Membrane Models: Neutron Reflectometry \& Molecular Simulation. Biophys J 2019, 116 (6), 1095-1104.

26. Heijne, G., The distribution of positively charged residues in bacterial inner membrane proteins correlates with the trans-membrane topology. The EMBO journal 1986, 5 (11), 3021-3027.

27. Jo, S.; Kim, T.; Iyer, V. G.; Im, W., CHARMM-GUI: a web-based graphical user interface for CHARMM. J Comput Chem 2008, 29 (11), 1859-65.

28. Brooks, B. R.; Brooks, C. L.; Mackerell, A. D.; Nilsson, L.; Petrella, R. J.; Roux, B.; Won, Y.; Archontis, G.; Bartels, C.; Boresch, S.; Caflisch, A.; Caves, L.; Cui, Q.; Dinner, A. R.; Feig, M.; Fischer, S.; Gao, J.; Hodoscek, M.; Im, W.; Kuczera, K.; Lazaridis, T.; Ma, J.; Ovchinnikov, V.; Paci, E.; Pastor, R. W.; Post, C. B.; Pu, J. Z.; Schaefer, M.; Tidor, B.; Venable, R. M.; Woodcock, H. L.; Wu, X.; Yang, W.; York, D. M.; Karplus, M., CHARMM: the biomolecular simulation program. J Comput Chem 2009, 30 (10), 1545614.

29. Wu, E. L.; Cheng, X.; Jo, S.; Rui, H.; Song, K. C.; Dávila-Contreras, E. M.; Qi, Y.; Lee, J.; MonjeGalvan, V.; Venable, R. M.; Klauda, J. B.; Im, W., CHARMM-GUI Membrane Builder toward realistic biological membrane simulations. J Comput Chem 2014, 35 (27), 1997-2004.

30. Jo, S.; Kim, T.; Im, W., Automated builder and database of protein/membrane complexes for molecular dynamics simulations. PLoS One 2007, 2 (9), e880.

31. Phillips, J. C.; Braun, R.; Wang, W.; Gumbart, J.; Tajkhorshid, E.; Villa, E.; Chipot, C.; Skeel, R. D.; Kalé, L.; Schulten, K., Scalable molecular dynamics with NAMD. J Comput Chem 2005, 26 (16), 1781-802.

32. Klauda, J. B.; Venable, R. M.; Freites, J. A.; O'Connor, J. W.; Tobias, D. J.; Mondragon-Ramirez, C.; Vorobyov, I.; MacKerell, A. D.; Pastor, R. W., Update of the CHARMM all-atom additive force field for lipids: validation on six lipid types. J Phys Chem B 2010, 114 (23), 7830-43.

33. Ben-Naim, A., On the driving forces for protein-protein association. J Chem Phys 2006, 125 (2), 24901. 
34. Jorgensen, W. L.; Chandrasekhar, J.; Madura, J. D.; Impey, R. W.; Klein, M. L., Comparison of simple potential functions for simulating liquid water. The Journal of Chemical Physics 1983, 79 (2), 926 935.

35. Steinbach, P. J.; Brooks, B. R., New spherical-cutoff methods for long-range forces in macromolecular simulation. Journal of Computational Chemistry 1994, 15 (7), 667-683.

36. Feller, S. E.; Zhang, Y.; Pastor, R. W.; Brooks, B. R., Constant pressure molecular dynamics simulation: The Langevin piston method. The Journal of Chemical Physics 1995, 103 (11), 4613-4621.

37. Martyna, G. J.; Tobias, D. J.; Klein, M. L., Constant pressure molecular dynamics algorithms. The Journal of Chemical Physics 1994, 101 (5), 4177-4189.

38. Andersen, H. C., Rattle: A "velocity" version of the shake algorithm for molecular dynamics calculations. Journal of Computational Physics 1983, 52 (1), 24-34.

39. May, S.; Kozlovsky, Y.; Ben-Shaul, A.; Kozlov, M. M., Tilt modulus of a lipid monolayer. Eur Phys J E Soft Matter 2004, 14 (3), 299-308.

40. Das, C.; Noro, M. G.; Olmsted, P. D., Simulation studies of stratum corneum lipid mixtures. Biophysical journal 2009, 97 (7), 1941-1951.

41. Venable, R. M.; Brown, F. L. H.; Pastor, R. W., Mechanical properties of lipid bilayers from molecular dynamics simulation. Chemistry and Physics of Lipids 2015, 192, 60-74.

42. Sonne, J.; Hansen, F. Y.; Peters, G. H., Methodological problems in pressure profile calculations for lipid bilayers. The Journal of Chemical Physics 2005, 122 (12), 124903.

43. Chodera, J. D., A Simple Method for Automated Equilibration Detection in Molecular Simulations. J Chem Theory Comput 2016, 12 (4), 1799-805.

44. Kollmitzer, B.; Heftberger, P.; Rappolt, M.; Pabst, G., Monolayer spontaneous curvature of raftforming membrane lipids. Soft matter 2013, 9 (45), 10877-10884.

45. Samuli Ollila, O. H.; Róg, T.; Karttunen, M.; Vattulainen, I., Role of sterol type on lateral pressure profiles of lipid membranes affecting membrane protein functionality: Comparison between cholesterol, desmosterol, 7-dehydrocholesterol and ketosterol. Journal of Structural Biology 2007, 159 (2), 311-323.

46. Chen, Z.; Rand, R. P., The influence of cholesterol on phospholipid membrane curvature and bending elasticity. Biophysical journal 1997, 73 (1), 267-276.

47. Feller, S. E.; Pastor, R. W., Constant surface tension simulations of lipid bilayers: The sensitivity of surface areas and compressibilities. The Journal of Chemical Physics 1999, 111 (3), 1281-1287.

48. Perly, B.; Smith, I. C.; Jarrell, H. C., Acyl chain dynamics of phosphatidylethanolamines containing oleic acid and dihydrosterculic acid: 2 H NMR relaxation studies. Biochemistry 1985, 24 (17), 4659-65. 


\section{TOC Graphic}

Symmetric bilayers

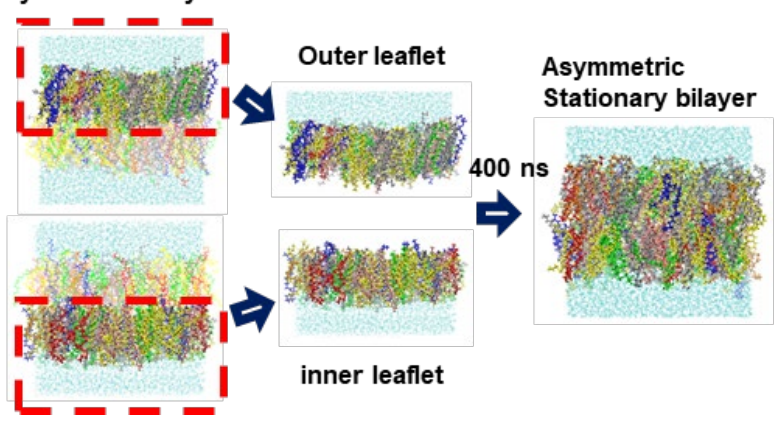

